\title{
Social Robots Helping People with Dementia: Assessing Efficacy of Social Robots in the Nursing Home Environment
}

\author{
Wendy Moyle ${ }^{1}$, Cindy Jones ${ }^{1}$, Marie Cooke ${ }^{1}$, Siobhan O’Dwyer ${ }^{1}$, Billy Sung ${ }^{1}$, Suzie Drummond ${ }^{1}$ \\ ${ }^{1}$ Griffith Health Institute, Centre for Health Practice Innovation, Griffith University, Brisbane, Australia \\ w.moyle@griffith.edu.au, c.jones@griffith.edu.au, m.cooke@griffith.edu.au, \\ s.odwyer@griffith.edu.au, b.sung@griffith.edu.au, s.drummond@griffith.edu.au
}

\begin{abstract}
This paper explores the challenges of testing the efficacy of social robots in the nursing home environment. The findings of two projects exploring the use of PARO and Giraff (a telepresence robot) are outlined. The challenges experienced by the researchers when undertaking a randomized controlled trial and using a case study design to test the social robots with people with dementia are examined. While randomized controlled trials are advocated, in early testing of the social robots the case study approach has the advantage that researchers can manipulate and adjust the intervention as issues arise. This can assist in the preparation of a protocol for testing in a randomized controlled trial at a later date.
\end{abstract}

Keywords: Dementia, Research Methods, Social Robots

\section{INTRODUCTION}

$\mathrm{T}$ HE population is ageing and as a result there are approximately 35.6 million older people globally and 298,000 Australians who have dementia [1]. Dementia is one of the major reasons why people enter nursing home care where approximately 104,400 people have dementia and account for around 53\% of the resident population [2]. Dementia is characterized by a progressive impairment of an individual's functioning and includes a decline in cognitive functioning, altered communication and depressed mood, which can often cause people with dementia to feel socially isolated and lonely. Furthermore, there can also be the emergence of agitated behavioral problems such as sleep-wake and rest-activity pattern disturbances.

Robots are increasingly being used to facilitate health and independent living in older populations. Cognitive impairment associated with dementia and the nursing home

The authors acknowledge Griffith University Office for Research and Griffith Health Institute for their equipment grant support that assisted with the purchase of these social robots and the Dementia Collaborative Research Centre- Carers and Consumers for financial support to conduct both of these projects.

Professor Wendy Moyle, Griffith Health Institute, Griffith University, 170 Kessels Road, Brisbane, QLD 4111, Australia. T: +617 37355526, F: +617 3735 5431, E: w.moyle@griffith.edu.au. environment adds to the complexity of testing the feasibility of robots for this population. This paper presents two vignettes to illustrate the benefits and challenges of social robots and then follows this with a brief overview of two pilot research projects involving social robots and discusses the challenges involved in researching the efficacy of social robots in a nursing home setting.

\section{BACKGROUND VIGNETTES}

\section{A. Thomas and PARO}

Thomas is 82 years old. He has moderate to late stage dementia and he has lived in a nursing home north of Brisbane, Australia since his daughter Susan found she could no longer meet his care needs. The care staff describes Thomas as a pleasant man who is no bother as, following his hygiene needs each morning; he sits in a lounge chair and waits patiently to be taken back to his room in the evening. He interacts and talks to no one and he has limited facial expression. The care staff believes there is little that they need to do for Thomas, as he seems content. His daughter, however, believes the care staff has limited time to spend with Thomas and he lacks stimulation and this has resulted in his current state of apathy. Susan volunteers Thomas to become involved in research involving a therapeutic emotional response robot - PARO. PARO, developed by Prof Shibata, a researcher at Japan's National Institute of Advanced Industrial Science and Technology, is a therapeutic pet-type robot, with the appearance of a baby harp seal and it is the size of a newborn baby [3]. It has tactile sensors and moves its tail and flippers, and opens its eyes when petted. Artificial intelligence software changes the robot's behaviors based on an array of sensors that monitor sound, light, temperature and touch. It responds to sounds, can learn its name and learns to respond to words its owner uses frequently. It can show emotions such as surprise, happiness and anger and will cry if it is not receiving sufficient attention. It produces sounds similar to a real baby seal and is active during the day and asleep at night.

As a decision was made to undertake the research facilitator training with Thomas (rather than including him as a participant in the study proper), the research team and several staff are present at the first training session. Thomas is presented with PARO and he immediately shows surprise, his facial expression suggesting he is unsure what 
PARO is. He gently pats PARO and PARO responds by looking up at him. He then places PARO onto his shoulder as one would with a small baby. Thomas looks content as he cuddles PARO tightly. He smiles as PARO shakes in response to his squeezing PARO tightly and he utters a quiet noise of surprise. His body and face again relax as he gently strokes PARO and PARO nuzzles into his neck. Later when the researchers go to take PARO from Thomas his face shows distress and he holds onto PARO's flippers in an attempt to retain PARO. The research team advises the research facilitator to gently state that PARO has to go home and to ask Thomas to say goodbye to PARO. Thomas releases his grip on PARO and looks directly at PARO's face as he says in a loud voice "Goodbye PARO". The eyes of staff watching are misty and become filled with tears, as this is the first time that they have heard Thomas speak in 2 years. Staff had perceived he could not speak and had stopped communicating with Thomas. This situation reminded staff of the importance of maintaining communication with people with dementia even when it appeared they had lost the ability to communicate.

This vignette illustrates the power of a robotic animal such as PARO (in this case in a very short time frame) to helping a person with dementia find their voice and express an emotional response that has the potential to change the way staff perceive and care for people with dementia, such as Thomas. Such a response may assist in improving quality of life and care of people with dementia.

While in Thomas' case the response was positive and continued to be so, PARO are expensive to purchase (approximately \$5,000US) and they require regular maintenance, which in our case in Australia requires us to send the PARO to Japan. As a result, we find ourselves asking how do we know if social robots and in this case PARO have a role in assisting social interaction, positive emotional response and improving quality of life of people with dementia? And importantly are robotic animals as or more effective than a human or live animal intervention?

The following vignette considers the telepresence robot Giraff.

\section{B. Joan and Giraff}

Joan is 83 years old. She has early to mid-stage dementia and lives in a nursing home in Brisbane, Australia. Her daughter Cindy lives close by to the nursing home and this offers her the opportunity to visit regularly. In the past Cindy used to call in each afternoon to see Joan but she stopped this activity as Joan was always asking to go home with her at the end of her visit and this distressed Cindy. As a result Cindy reduced her visits to once a week at the weekend when she had more time to cope with disengaging from Joan. However, Joan became agitated when Cindy's visits became less regular and she was concerned that she was not seeing Cindy each day. Joan was therefore referred to the research team as a potential participant to 'test' the feasibility of linking with her daughter Cindy through a telepresence robot. Telepresence robots allow the user to move virtually such as in a nursing home environment by using an external computer to remotely control the robot. The family and resident are able to see and hear each other through two-way live video via the robot's screen and microphone. The team in this situation tested a Giraff telepresence robot.

The Giraff robot [4], affectionately called Gerry by the research team, was introduced to Joan and Cindy and Cindy was trained and provided with software to download, with the aim that Cindy could connect with Joan three times weekly via the telepresence robot Gerry. Joan and Cindy both responded positively to the opportunity to see each other via the telepresence robot and for Joan to be able to show Cindy items she was interested in within the nursing home. Joan's immediate response when she first viewed Cindy's face on the robot screen was "How did she get in there"? Interaction via the robot helped to comfort Joan as this offered her the opportunity to see and hear from Cindy more frequently. Furthermore, Cindy was relieved that Joan was not walking her to the door of the nursing home and asking to go home with her. The care staff also commented that Joan was more settled and they enjoyed seeing her laughing and communicating with her daughter via Gerry.

Although this vignette demonstrates a positive result, there were a number of challenges posed by the technology for the users. For example, the quality of the Internet connection posed challenges in connecting and maintaining connection via the robot software; the robot was built in Sweden, a much cooler climate than Australia and warmer Australian temperatures often resulted in the robot overheating. The family member was often challenged by the task of remotely navigating the robot into the resident's room via controls on their computer while avoiding obstacles within the nursing home. However, the biggest challenge was in overcoming negative staff attitudes toward robots and convincing them that the time we were asking of them to move the Giraff robot and to help with the connection would be beneficial to the resident and family and could potentially have a positive impact on their provision of care.

When putting together the proposal for this research we found ourselves asking what data we need to assist with understanding the feasibility of using such a robot. This vignette illustrates the opportunities that telepresence robots can offer to older people with dementia and their family carers. Innovative robotic technologies promise to enhance the life of older people, carers and staff. There are a growing number of robots available that may offer additional opportunities to this group but the challenge is in how to determine the efficacy of robots and the methods to determine their use.

\section{CHALLENGES OF TESTING EFFICACY OF SOCIAL ROBOTS: PARO}

The researchers have been testing several animal and telepresence robots to determine their efficacy and feasibility for use with people with dementia living in nursing home settings. The most popular research design being used by the team to test efficacy are randomized controlled trials (RCTs) and in initial piloting for feasibility, a case study approach. The remainder of this paper will outline the challenges of testing the efficacy and feasibility of two social robots; PARO and Giraff. 


\section{A. PARO Research}

PARO, a therapeutic emotional response robotic seal, is used in a number of countries as a means of inducing relaxation, socialization, and motivation in older people. Although there are several PARO in Australia they tend to be used sporadically as a means of reducing agitated behaviors displayed by people with dementia. However, our discussions with people who have purchased PARO found PARO were more than likely to be kept long-term in storage as the cost of the PARO induced concern that regular use would damage the PARO and there were also concerns about the cost of maintenance. Our review of the literature prior to writing the research proposal failed to identify rigorous research using PARO. The researchers received funding from the Dementia Collaborative Research Centre- Carers and Consumers [5] to conduct pilot research on the efficacy of PARO with the aim of producing findings that, if deemed to be positive, could be used to seek funding for a larger study.

Findings from a pilot study conducted by members of our research team [6], set out to compare the effect of therapeutic robots (PARO) with interactive participation in a reading group on emotional response in people living with dementia in a nursing home setting. This study adopted a randomized controlled crossover design with repeated measures across three time periods (baseline, mid-point, end of intervention). The study was conducted in one nursing home setting. The study sought to recruit 16 residents with mid to late stage dementia, as this is the time in the dementia trajectory when most disruptive behaviors occur. The nursing home operator was keen for their staff to learn from the study and negotiated for the facility activities officer to be trained as the facilitator of the study intervention and control activities. The activities officer was a degree qualified arts therapist and had a number of years experience in providing therapeutic activities with people with dementia.

Family members of 18 residents living in long-term care with a diagnosis of mid to late stage dementia agreed for their family member to be recruited for the PARO study. Participants were randomized to treatment order and they participated in the group PARO intervention (45 minutes duration), three times a week for five weeks or an interactive reading group for the same time period. They then crossed over into the opposite intervention after a three-week washout period. The intervention and control group activity were facilitated by the trained activities officer and undertaken within a group of nine participants in one section of the care facility. Both the intervention and control protocol were designed around the four concepts of: (i) discovery; (ii) engagement, (iii) social interaction, and (iv) touch. One PARO was used in weeks 1 to 3 and in weeks 4 and 5 a second PARO was introduced with the aim that this would allow each individual more individual time to spend with PARO.

Participants were assessed three times using the Quality of Life in Alzheimer's Disease (QOL_AD) scale [7], Observed Emotion Rating Scale (OERS) [8] and Revised Algase Wandering Scales (RAWS) [9]. PARO was found to have medium to large positive influences on two of the scales: QOL-AD (0.6 to 1.3) and OERS-Pleasure score (0.7) and these scores were higher in the PARO group when compared with the reading group [6]. Staff indicated that participants in the PARO group displayed less anxiety than those in the Reading group and this was confirmed by video analysis of group sessions. Videos were analyzed using Noldus Observer XT software [10]. Participants were also found to display significantly longer periods of positive than negative or neutral engaging behaviors during the PARO sessions.

\section{B. PARO efficacy and feasibility}

The findings from this pilot study indicate that PARO is a social robot that is feasible for use with people with midto late-stage dementia and may have a place in improving their mood and social interaction. The characteristics of people with dementia and the nursing home environment, however, can add to the complexity and challenge of testing the efficacy and feasibility of PARO. In particular conducting randomized controlled trials and the cost of such trials also reduces the likelihood of such a rigorous design being used where research team expertise in clinical trials and adequate funding is not available.

\section{Challenges of RCT design}

There were a number of challenges encountered in this project and included the following:

Recruitment: To meet the concerns of the research ethics board, we relied on the facility staff to identify residents who meet the study eligibility criteria and for them to provide informed consent materials to residents' families for proxy informed consent. It proved challenging for staff to identify participants who met the eligibility criteria and this frequently resulted in the team being introduced to families and residents who were not eligible to participate in the study. Not only was this time consuming, but also families and residents were often disappointed when they were deemed ineligible to participate.

Logistical issues: The need for consistency during the implementation of the intervention and control are essential. However, the nursing home environment is often not conducive to rigorous research where the intervention is given in a precise manner and where the variables need to be controlled. [11]

Finding an environment to undertake the research was challenging as the lounge areas that could readily fit the number of participants were all open areas and on view and accessed by other residents. This resulted in PARO being covered with a blanket when taken through the nursing home to the lounge so that other residents didn't follow and want to be involved. In addition the researchers screened the lounge area. As the screens were mobile this resulted on occasion in some residents pushing through the barriers to enter the room during the intervention or participants leaving the room when they become restless.

Some participants had outbursts of disruptive behavior during the intervention, in particular when PARO was focused on the group of participants rather than the individual. Such behaviors had the potential to influence the mood of the group as well as the potential benefit of PARO.

Videoing the session was also challenged by the shape of the room and finding a place we could readily place the video cameras that was not intrusive. In addition, maintaining session attendance was challenging as illness, 
doctor's appointments, family visits and other activities within the facility often disrupted attendance.

Fidelity of intervention: The facility manager wanted the research team to employ the facility diversional therapist (DT) to run the PARO program as he was an arts therapy and dementia expert and knew the residents and their abilities. Although challenged by this request two members of the team trained the DT to provide the intervention and the team undertook regular spot checks as the intervention proceeded to ensure the standardized procedure was maintained. In spite of the training the DT was challenged by the standardized intervention and the video recordings suggested that he never really looked comfortable facilitating the intervention. Sometime after the research the DT confessed that he had perceived the intervention would not be of benefit to the residents but over time he was surprised to see the benefits of PARO. Although the video recordings and spot checks confirmed that he maintained the standardized procedure his initial negative attitude towards PARO and his discomfort with the intervention had the potential to influence the effect of the intervention.

Outcome measures and data collection: Deciding upon the instruments and scales to use in any study can be a difficult task. Three main outcome measures that meet the aims of the project were employed. These measures were also chosen for their reliability and validity and because they varied in methodological approach to data collection. For example, they enabled data to be collected via observation, self-report and proxy report. As the majority of participants had late stage dementia the self-report data was challenging for a number of participants and this resulted in missing data. Without the proxy and observation measures there would be limited data collected and able to be analyzed.

\section{Consideration needs to be given to the following}

When planning a RCT study using a psychosocial intervention, such as PARO the following considerations need to be explored.

- Are there enough potential participants available who will meet the study eligibility criteria?

- Does staff have the knowledge/skill to select potential participants for the study?

- Is there a suitable environment in which to undertake the intervention and does getting to the environment pose problems for getting participants to the room?

- What equipment is needed and can it be managed within the environment, i.e. video cameras?

- Is a person/s required to conduct the intervention? What are the knowledge/skill/attitudes of intervention staff and how will you ensure they conduct the standardized intervention?

- What factors might influence participant attendance and can these be worked around?

- Which outcome measures will be appropriate for the potential population?

\section{Challenges of TeSTing EFFICACY OF SOCIAL ROBOTS: GIRAFF}

Giraff is a remotely controlled, mobile, human-height, telepresence robot. It is manufactured by Technologies $A B$ in Sweden and is equipped with a videoconferencing system that includes a video camera, LCD screen, speaker and microphone [4]. Using this videoconferencing system, families can 'virtually' visit people with dementia engaging in two-way conversations, with their face appearing on a 'life size' video screen. This enables, in this case, the family member and the person with dementia to view each other and also allows the family member to view the nursing home environment. While there are many potential situations in which telepresence robots could be used to support older people and promote social interaction, the newness of this technology means that there are only a few studies that have tested the feasibility and the effectiveness of this particular technology in an older population and we are not aware of any of these studies being focused on people with dementia. The researchers received funding from the Dementia Collaborative Research Centre - Carers and Consumers to conduct the research [12].

\section{A. Giraff Research}

Using a case study design [13] to allow individuals, practices and the Giraff experience to be described over time, this study aimed to gather initial pilot data to explore the feasibility of using the Giraff in nursing home care to encourage communication between family living in the community and the person with dementia. The method involved five research triads each comprising of: one resident with dementia living in a long-term care facility; a family member; and up to two members of the care staff team who assisted in the set up of the robot.

The experiences of the Giraff were explored through video observation of the family-resident communication and semi-structured interviews with individual members of each dementia triad. Preliminary data analysis demonstrated that the Giraff has a place in assisting people and their families to connect and through such a connection there is the potential for an improvement in the emotional state of the person with dementia. Furthermore, the Giraff experience offered the opportunity for staff to view the positive outcomes of keeping people with dementia active within the nursing home setting and connected with family on a regular basis. Families and staff expressed interest in keeping the Giraff at the nursing home at the end of the study.

\section{B. Giraff feasibility}

Although this research encouraged a lot of media interest and the team were excited by the outcomes of the trial, the many challenges of the technology suggests that the Giraff robot needs a little more refinement before it can be readily integrated into nursing home care within Australia.

\section{Challenges of Giraff Research}

There were a number of challenges in conducting this research, with the majority of issues being related to 
connectivity failure either due to poor Wi-Fi connection or the software system being out of action for a short time, and the Australian summer temperatures as well as the additional equipment we added to Giraff causing an overheating of Giraff. In addition the majority of staff felt unable to help the resident or family member to connect via Giraff and they felt they did not have the time to learn this skill. Furthermore, families were often challenged by the task of remotely 'driving' Giraff to the residents' bedside, as the visual field they saw on their computer screen did not provide accurate depth perception and this often resulted in the Giraff bumping into objects or being placed at a distance from the resident. Although the characteristics of the robot and the environment added to the complexity and challenge of this research, the case study approach allowed the team to overcome such challenges as the intervention was individualized. For example, if the connection posed a problem an alternative time was set up for another connection. Procedure manuals written by a member of the research team as well as training ensured the family and staff were prepared for the intervention to take place. While the case study approach does not have the rigorous features of a RCT design, in this situation where we were testing the feasibility of the Giraff this was most appropriate as it allowed the team to readily understand the feasibility of the robot and to fix problems as they occurred.

\section{Consideration needs to be given to the following}

When planning case study research using technology such as Giraff the following considerations need to be explored.

- Is there interest in undertaking the research from staff, families and residents?

- Is the environment suitable to undertake the research?

- Is the telecommunication system within the environment adequate to support the technology?

\section{RECOMMENDATIONS}

Design features can impede the use of robots in the nursing home environment. Our team has been testing a number of robots. The team suggests there are features robotic designers need to consider when developing robots for use for older people and in particular people with dementia. Designers must understand the perceptual, motor control and cognitive capabilities of users and, in this case, their carers who are the people often responsible for assisting with using the robot.

The robots we have been testing arrive either with sophisticated manuals explaining in detail the robot set up; limited instruction manuals; or manuals translated from a foreign language into English. In every case these need re-writing. The manuals can sometimes prove to be too sophisticated for the average user. The ageing process, staff and carers with limited education, and the dementing process may also result in the need for lay instructional manuals that enable users to effectively interact with the social robots. Prior to testing the robots, time is often spent by the research team in writing new instructional manuals alongside our research protocols, using photographs, diagrams and process steps that are tested prior to commencing the research. To assist with access and use there have also been times the researchers have attached cue cards to the robot to remind the user of the steps to follow.

Robots must be well designed to work within the environment they are intended. The importance in this case is in designers working with social scientists, health professionals, and people for which the robot is intended, so that the approach is user-centered to ensure the robot meets a need, is reliable in accomplishing the tasks to meet the need, is concerned with the limitations of the audience for which it is intended, is an appropriate size and shape that can fit within the intended environment, and is made of materials and colors that look appealing. The look and appeal of robots is an area that often is ignored by developers and yet our work constantly convinces us that older adults in particular want a robot that looks friendly, offers tactile comfort (even if this is not the main purpose of the robot), and has features such as buttons and screens that can easily be manipulated with arthritic hands, impaired vision and cognitive decline.

\section{CONCLUSION}

Older adults including people with dementia and their carers are willing to use and to try robotic technologies. To optimize the use of new robots developers need to work with social scientists, health professionals and the people for whom the robots are intended, so that the robots developed meet the intended needs. Furthermore, robots that have aesthetic appeal and can be used by older people with physical and mental frailty will assist in the adoption of such technologies. Future research in the use of social robots must address the limitations of the current research. Therefore, the researchers advocate such research must use gold standard randomized controlled trial methods and alongside this approach also collect qualitative interview and observational data. A case study approach is of important use in the initial piloting or set up of the study protocol.

\section{ACKNOWLEDGMENT}

Acknowledgments are given to the following two aged care organizations: RSL (QLD) War Veterans' Homes Ltd and Churches of Christ for their support. Further acknowledgement is given to the Dementia Collaborative Research Centre - Consumers and Carers (QUT) for funding to undertake the research and to Griffith University for infrastructure funding to support the purchase of these robots.

\section{REFERENCES}

[1] Australian Institute of Health and Welfare (2012) Dementia in Australia. Cat no. AGE 70, Canberra, AIHW

[2] Australian Institute of Health and Welfare (2011) Dementia among aged care residents: first information from the Aged Care Funding Instrument. Aged care statistics series no. 32. Cat. no. AGE 63, AIHW, Canberra

[3] PARO http://www.parorobots.com/index.asp accessed 8th April 2013

[4] Giraff Robot http://www.oru.se/ExCITE/Part-3/. Accessed 8 April 2013

[5] Moyle W, Cooke M, Beattie E, Jones C, Cook G, \& Klein B (2010). The effect of PARO on social engagement, communication and quality of life (QOL) in people living with dementia in residential 
care, research funding received from Dementia Collaborative Research Centre-Carers and Consumers, QUT

[6] Moyle W, Cooke M, Beattie E, Jones C, Klein B, Cook G, Gray C (2013). Exploring the effect of companion robots on emotional expression in older people with dementia: A pilot RCT. J Geron Nurs, 03/2013, DOI:10.3928/00989134-20130313-03.

[7] Edelman P, Fulton BR, Kuhn D, Chang CH (2005) A comparison of three methods of measuring dementia-specific quality of life: Perspectives of residents, staff, and observers. The Gerontologist 45(1): 27-36

[8] Lawton MP, Van Haitsma K, Klapper J (1999) Observed emotion rating scale. J Ment Hlth Aging 5(1): 69-81

[9] Algase DL, Beattie ERA, Bogue EL, Yao L (2001) The Algase Wandering Scale: initial psychometrics of a new caregiver reporting tool Am J Alzheim Dis Other Dem 16(3): 141-152.

[10] Noldus Observer XT Software.

http://www.noldus.com/human-behavior-research/products/the-obse rver-xt

[11] Murfield J, Cooke M, Moyle W, Shum D, \& Harrison S (2011) Conducting randomised Control Trials with older people with dementia in long-term care: Challenges and lessons learn. International Journal of Nursing Practice, 17, 52-59

[12] Moyle W, Jones C, Cooke M, O’Dwyer S, Sung B (2012) Piloting a "Telepresence Robot": feasibility and impact on the person with dementia and their family, research funding received from Dementia Collaborative Research Centre-Carers and Consumers, QUT

[13] Yin YK (2009) Case study research: Design and methods Sage Publications, California 